\title{
Silicon Photomultiplier Use in Particle Astrophysics and Heliophysics Missions
}

\author{
Jason T. Link ${ }^{* 1,2}$, Y. Akaike ${ }^{1,2}$, W.R. Binns ${ }^{3}$, R.G. Bose ${ }^{3}$, T.J. Brandt ${ }^{1}$, J.H. Buckley ${ }^{3}$, \\ S.O. Cannady ${ }^{1}$, G.A De Nolfo ${ }^{1}$, P.F Dowkontt ${ }^{3}$, J.J. Du Monthier ${ }^{1}$, Z.D. Hughes ${ }^{3}$, I. \\ Liceaga-Indart $^{1,4}$, M.H Israel ${ }^{3}$, J.F Krizmanic ${ }^{1,2}$, A.W. Labrador ${ }^{5}$, R.A Mewaldt ${ }^{5}$, J.G. \\ Mitchell $^{1,6}$, J.W Mitchell ${ }^{1}$, S. Nutter ${ }^{7}$, B.F Rauch ${ }^{3}$, K. Sakai ${ }^{1,2}$, M. Sasaki ${ }^{1,8}$, E.C \\ Stone $^{5}$, G. Suarez ${ }^{1}$, T. Tatoli ${ }^{1,2}$, C.J. Waddington ${ }^{9}$, M.E. Wiedenbeck ${ }^{10}$ \\ ${ }^{1}$ NASA Goddard Space Flight Center, Greenbelt Maryland, 20771, USA. \\ ${ }^{2}$ CRESST-UMBC, Baltimore, Maryland, 21250, USA. \\ ${ }^{3}$ Washington University in St. Louis, St. Louis, Missouri, 61630 USA. \\ ${ }^{4}$ The Catholic University of America, Washington DC, 20064, USA. \\ ${ }^{5}$ California Institute of Technology, Pasadena, California, 91125, USA. \\ ${ }^{6}$ George Washington University, Washington DC, 20052, USA. \\ ${ }^{7}$ Northern Kentucky University, Highland Heights, Kentucky, 41099, USA. \\ ${ }^{8}$ CRESST-UMCP, College Park, Maryland, 20742, USA. \\ ${ }^{9}$ University of Minnesota, Minneapolis, Minnesota, 55455, USA. \\ ${ }^{10}$ NASA Jet Propulsion Laboratory, Pasadena, California, 91109, USA. \\ E-mail: Jason.T.Linkenasa.gov
}

\begin{abstract}
Silicon Photomultipliers (SiPM) are increasingly being used in ground-based astrophysics experiments as a replacement for Photomultiplier tubes (PMTs). These compact low power, rugged sensors are also well suited for space applications and have an added advantage over PMTs in that they do not require high-voltage bias. Efforts are underway at NASA Goddard and our collaborators to develop large-area arrays of SiPMs as the readout for the upcoming funded CubeSat missions such as BurstCube and Terrestrial RaYs Analysis and Detection (TRYAD) and also larger missions including the ultra-heavy cosmic-ray Heavy Nuclei Explorer (HNX) experiment and the gamma-ray Advanced Particle-astrophysics Telescope (APT) experiment. Our team has recently tested SiPMs in a heavy ion beam at CERN. The primary beam consisted of lead ions with energy of $150 \mathrm{GeV} / \mathrm{nuc}$. SiPM hardware was exposed to both a pure and fragmented $\mathrm{Pb}$ beam allowing us to look at ions from $\mathrm{Z}=1$ to $\mathrm{Z}=82$. We report in this paper on direct comparisons with the performance of Hamamatsu R1924A photomultipliers in addition to examining the signals (and subsequent effects) from direct exposure to a lead beam.
\end{abstract}

36th International Cosmic Ray Conference -ICRC2019-

July 24th - August 1st, 2019

Madison, WI, U.S.A.

${ }^{*}$ Speaker. 


\section{Introduction}

Silicon Photomultipliers (SiPMs) consist of an array of photodiode elements operating in geiger mode. They are biased several volts higher than their breakdown voltage (typically 2-5 volts). Incident photons are adsorbed in the silicon creating a electron-hole pair. The bias creates an electric field that sweeps these charge carriers towards the anode (hole) or cathode (electron). In geiger mode, the bias accelerates the charge carriers so that secondary charge pairs are created through impact ionization, which creates gain in the SiPM. The photodiode elements include an integrated resistor, which quenches this avalanch after tens of nanoseconds, and are read out in parallel. The total signal of the SiPM is propotional to the number of photodiode elements that fire from being struck by a photon.

SiPMs are replacing photomultiplier tubes (PMTs) in modern particle and photon detectors and instruments. SiPMs have significant advantages over PMTs including a lack of a glass vacuum tube and small size leading to a more compact and robust packaging. In addition a SiPM requres a bias voltage under $60 \mathrm{~V}$ versus $1000 \mathrm{~V}$ or more for most PMTs. The high voltage needed to bias the PMTs is harder design and operate requiring special power supplies, cabling, potting and additional environmental testing. Working with the high voltages required for PMTs is particularly challenging for scientific balloon instruments which fly at atmospheric pressures (3-5mbar) near the minimum of the Paschen Curve. A major disadvanage of SiPMs is their greater temperature sensitivity. The gain of the SiPM depends on the difference between the bias voltage and breakdown voltage. Temperature variations can lead to significant gain variations in the device and the dark current of the SiPM will also increase with temperature.

Several instrumental groups at NASA Goddard are involved in efforts to utilize SiPMs in space and near-space applications as a replacement for PMTs in astroparticle detectors. Two missions in production at Goddard are Cube-Sat gamma-ray instruments: Terrestrial RaYs Analysis and Detection (TRYAD), which is designed to observe terrestrial gamma-ray flashes, and BurstCUBE, which is designed to detect and measure gamma-ray bursts. Both of these Cube-Sat missions are currently funded and under development. SiPMs are also being considered for use in the Cherenkov lightboxes of the the Heavy Nuclei Explorer (HNX), which, measures the energy and elemental composition of elements up through the actinides to the end of the periodic table and the Washington University lead Advanced Particle-astrophysics Telescope (APT), which, measures gamma-rays from $1 \mathrm{MeV}$ to $50 \mathrm{GeV}$ with an order of magnitude greater than Fermi, and potentially be able to measure cosmic rays.

\section{CERN 2018}

The NASA Goddard High Energy Cosmic Ray Group was given time to test ultra-heavy particle detector technology at CERN in 2018 from November 19-Dec 2. Testing occurred in the H8 beam line at the North Area of the CERN SPS. During this period the team was able to expose detectors to a $\mathrm{Pb}$ beam with energy of $150 \mathrm{GeV} / \mathrm{n}$. In addition to the "pure" lead beam the team also looked at a fragmented beam with $\mathrm{A} / \mathrm{Z}$ selected to 2.0,2.2 and 2.4 to explore and understand the response of detectors to all charges up to $\mathrm{Pb}(\mathrm{Z}=82)$. The primary goals of this test was to demonstrate the resolution and response of silicon strip detectors and SiPMs to charges between 
5 and 82. Results for the silicon strip detectors are reported elsewhere at this conference [1]. A secondary goal was to expose SiPMs directly to the primary and fragmented beam to determine if there was any significant damage done to these photosensors by the heavy ions. Additionally, a prototype APT detector was built by a team at Washington University and exposed to the test beam. Data from this exposure will allow the APT team to understand the response of the detector to a charged particles and give a better understanding of the sensitivity and response of a full scale instrument. A diagram of the arrangment of detector modules for most of the test is shown in Figure 1. The SiPM exposure box was not present in the beam for the full period of testing and one of the Caltech Silicon Detector Systems (CIT Si 3 and 4) was placed behind the APT prototype for part of the test to create a dataset that could be corrected for interactions of particles passing through all of the detector modules.

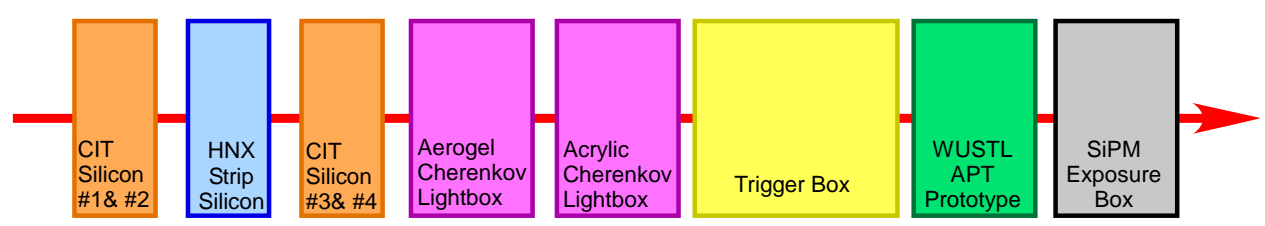

Figure 1: Detector Configuration for 2018 CERN beam test. The 3 boxes closest to the beamline exit contain silicon detectors. Two of these boxes contained calibration detectors from Caltech while the third holds the prototype HNX strip detectors. Behind these boxes are two Cherenkov lightboxes, one with an acrylic radiator and one with a aerogel radiator. Light from these boxes is detected by both PMTs and a SiPM array. A trigger box resides behind the Cherenkov light boxes. Behind this is an APT prototype detector from a team at Washington University in St. Louis. The final enclosure containes a SiPM board with SiPMs aligned to be directly in the beam path.

The Silicon and Trigger Box were identical to those used in the 2016 CERN experiment and are described elsewhere [2]. The acrylic and aerogel lightboxes built from aluminum with a Cherenkov radiator installed perpendicular to the beam and a pair of three inch cutouts on the sides to accomodate photosensors to look into the light box. One cutout had a piece of delrin with two R1924A PMTs installed while the cutout across from it other had a piece of delrin machined to hold an electronics board with thirty SiPMs installed. This board was designed by the Code 672 Energetic Particle Lab (EPL) at NASA Goddard. The EPL board has a configurable design to allow it to be fabricated to support any number of SiPMs in a custom arrangement. The board provides summing and shaping of the signal from the SiPMs installed in order to pass it to a data acquisition system. The acrylic radiator was a $21 \times 15 \times 1.4 \mathrm{~cm}$ piece of Spartech/ Polycast acrylic with a bis-MSB wavelength shifter dye added $(25 \mathrm{mg} / \mathrm{L})$ and has an index of refraction $n=1.49$. The aerogel radiator was a piece of aerogel $21 \times 15 \times 3 \mathrm{~cm}$ with $\mathrm{n}=1.04$ index of refraction. A picture of the acrylic lightbox is shown in Figure 2.

The data acquisition system consisted of spares from the SuperTIGER experiment, and included front end electronic (FEE) boards that digitize the signal, an IO board that enables triggering and data collection, and a flight computer [3]. This data acquisition system required a beam trigger that was delayed by $\approx 3$ microseconds after a beam particle's passage through the beamline detectors. The trigger needed to be moderately efficient for charges $1 \leq \mathrm{Z} \leq 82$, corresponding to the range of fragments possible from a lead beam. The beam rate was $\approx 1 \mathrm{kHz}$, so pileup was 


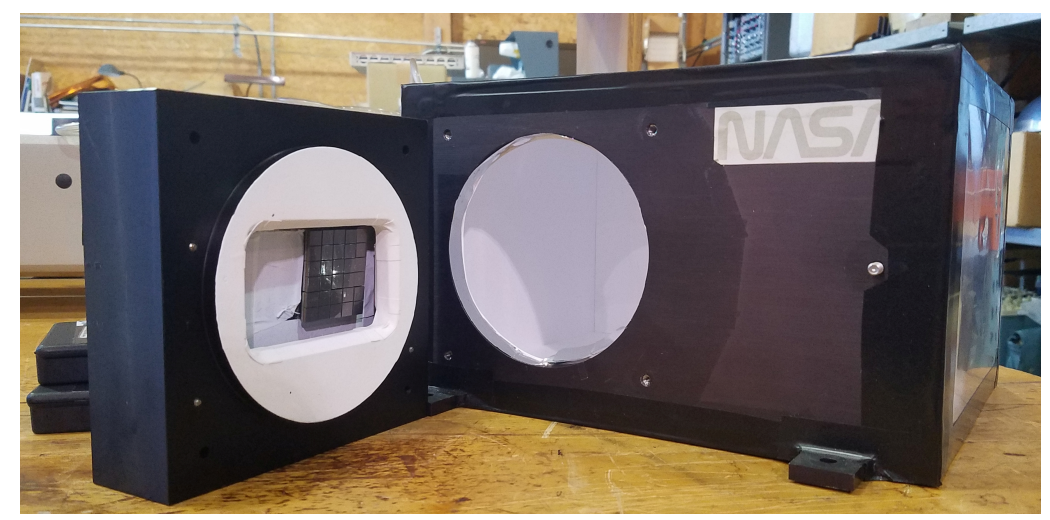

Figure 2: Photo of the Cherenkov Lightbox used in the CERN 2018 beamtest and the EPL SiPM board with 30 S14160 SiPMs installed

not an issue and fast timing was not a critical design parameter. The trigger required a coincidence between the Hamamatsu R1924 PMTs on the two integrating Cerenkov boxes (acrylic and aerogel) after the PMT signals were split between the acquisition system and the trigger. The trigger signals were inverted then discriminated $(\approx 1$ Volt threshold on a LeCroy 821 , with 100 ns widths) and a coincidence generated by a LeCroy 622 logic module. The NIM-level signal started a gate generator set to a 1 microsecond width that was converted by a LeCroy 688AL level convertor to TTL. The TTL gate went to a Stanford DG535 gate generator set to generate the trigger TTL pulse after a 3 microseconds delay.

\section{Performance Characterization of SiPMs and PMTs}

As part of our CERN test we compared the dynamic range and response of Hamamatsu S14160-6050HS SiPMs with Hamamatsu R1924A photomultipliers in the Cherenkov lightboxes. Two R1924A PMTs with a total active photocathode area of $760 \mathrm{~mm}^{2}$ were installed looking into the lightbox perpendicular to the beam across from the thirty S14160 SiPMs. The SiPM array has a total active area of $1080 \mathrm{~mm}^{2}$. The PMTs are spares from the SuperTIGER experiment, and run at $950 \mathrm{~V}$ from an external supply. The S14160 SiPMs were biased to $40.8 \mathrm{~V}$ and installed on an EPL SiPM board modified so its shaping and amplification were compatible with the SuperTIGER readout system.

For our analysis we examined events taken during the run when two of the Caltech silicon detectors were located behind the APT detector. This enabled an interaction cut of the data to be made using the Caltech silicon detectors requiring charge consistency between the front and back silicon detector systems. Additionally, only data from the acrylic lightbox was considered as the charge data from the aerogel lightbox was quite poor suggesting a contaminated radiator.

In Figure 3 we plot the summed response of the average of the two R1924 PMTs versus the summed response of the $30 \mathrm{SiPMs}$ in the acrylic lightbox. One can see from the figures on the right that there are clearly resolved charge distributions on the crossplot from Boron $(Z=5)$ all the way to Lead $(Z=82)$. This confirms that both the SiPM and PMTs have the required dynamic range and response to measure all elemental charges between $\mathrm{Z}=5$ and $\mathrm{Z}=82$. 

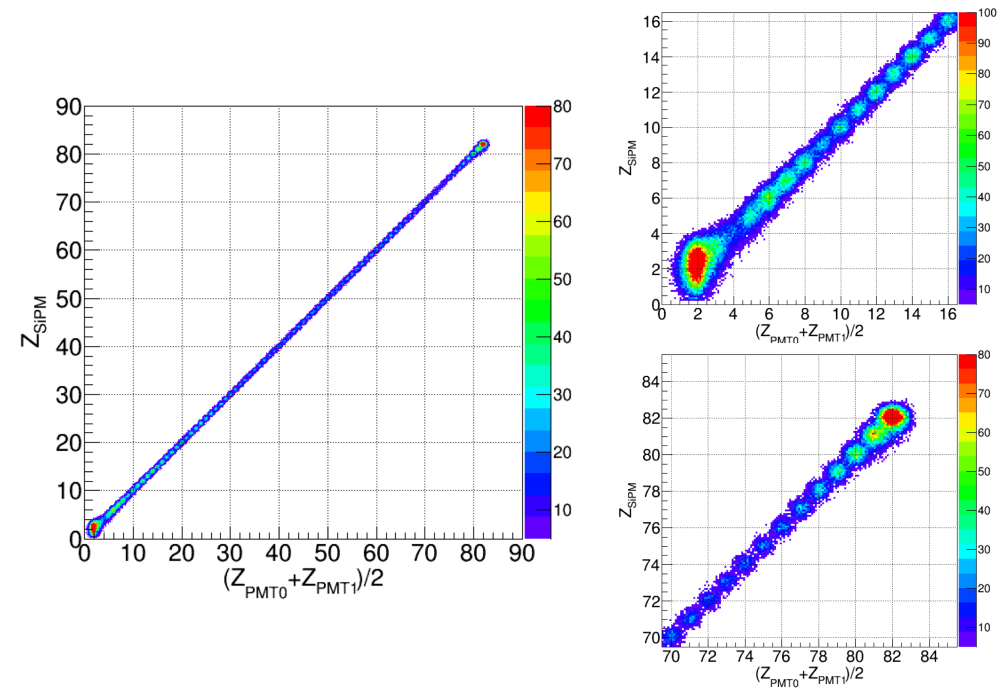

Figure 3: Comparison of the response of signal from an Hammamatsu R1924A PMT and an array of S14160 SiPMs. The plot on the left is a crossplot of charge as determined by the photosensor based on histograms versus the CIT Silicon detectors with interactions removed. The plots on the right are expanded views of the low charge regime and high charge regime of the plot to the left.

A comparison of the charge resolution of PMTs and SiPMs was obtained by fitting a gaussian to the lead peak and converting the ACD scale to MIPs. A linearity correction was made between our "pure" lead run and each of the fragmentation runs. Charge was calculated as $Z=\operatorname{sqrt}($ MIP) yielding the charge histogram show in Figure 4. In Figure 5 we show a comparison of the charge resolution of the averaged sum of the individual Hammamatsu PMTs, the summed average signal of the PMTs and the SiPMs. The charge resolution for the SiPM and the summed PMTs are very similar, though the SiPM generally have a slightly better resolution over the charge range.

\section{Effects of Heavy Ions on SiPMs}

A concern about using SiPMs in high energy and astrophysical applications is how radiation affects the operation and performance of the photosensor. There has been extensive studies of SiPMs for light ions, for instance by Musienko et al for the CMS [4]. In their studies, SiPMs were exposed to $62 \mathrm{MeV}$ protons at fluences up to $1 \times 10^{12}$ protons $/ \mathrm{cm}^{2}$ at the ULC proton cyclotron. They found that the exposed SiPMs had significant increases in dark current and noise and one SiPM had a significant signal gain reduction which was found to be primarily due to a breakdown voltage shift after it was irradiated. The SiPM did still function during and after irradiation, however the increase in dark current and noise require a higher noise threshold to be set which leads to a lower sensitivity and reduced dynamic range.

For astrophysical applications we should also consider if the larger particles of heavy ions can cause significant damage to the SiPM photoelements. Our team performed a preliminary exposure test at CERN in 2016 of a SensL MicroFC-SMA-60035 in a $150 \mathrm{GeV} / \mathrm{n} \mathrm{Pb}$ beam with A/Z=2 as part of a test of new silicon strip detectors for the HNX experiment. We found that exposure to 6400 events did not have any signifcant effects [2]. Due to our readout and trigger system and the 

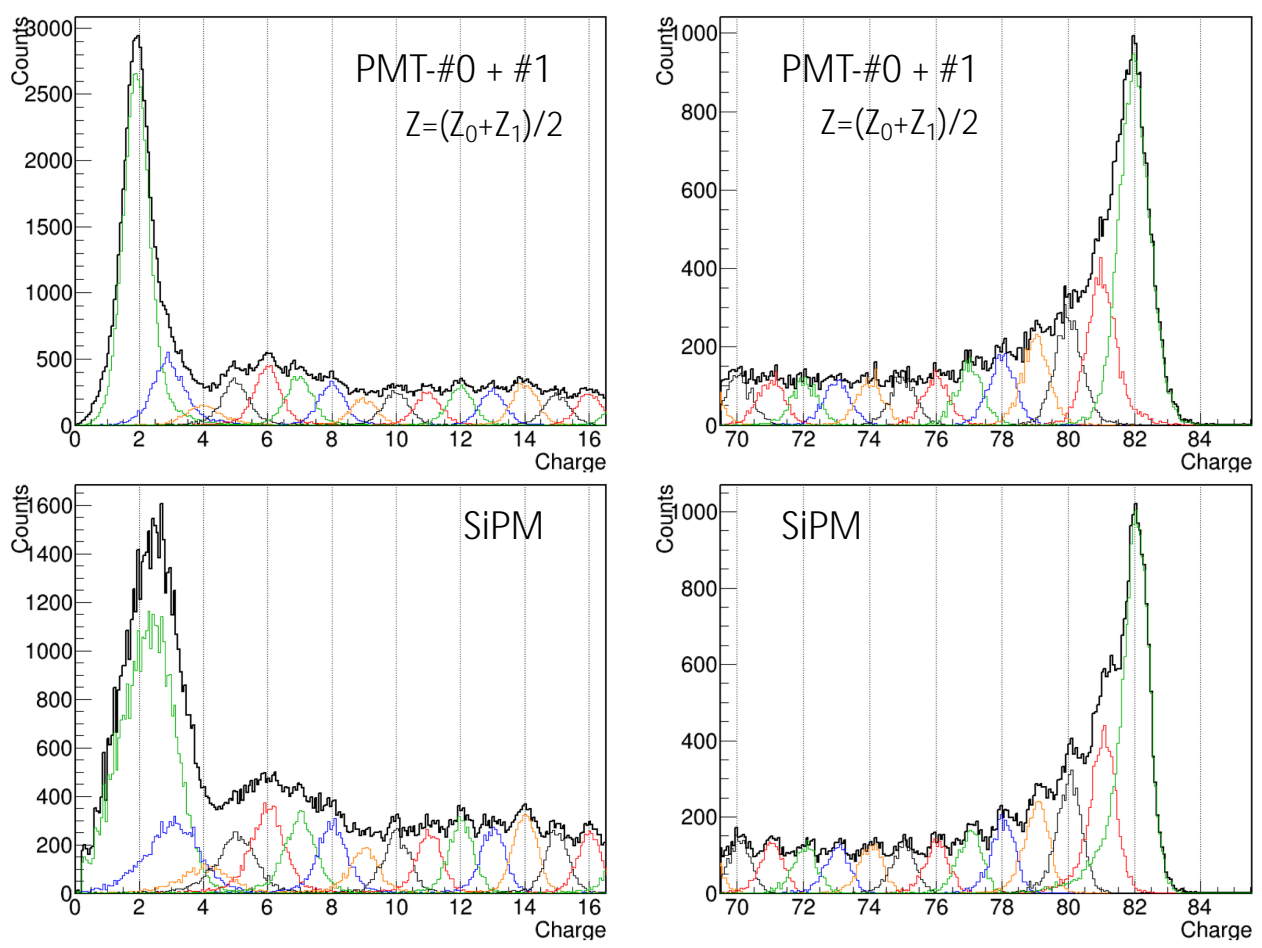

Figure 4: Charge histogram of the R1924 PMTs and S14160 SiPMs
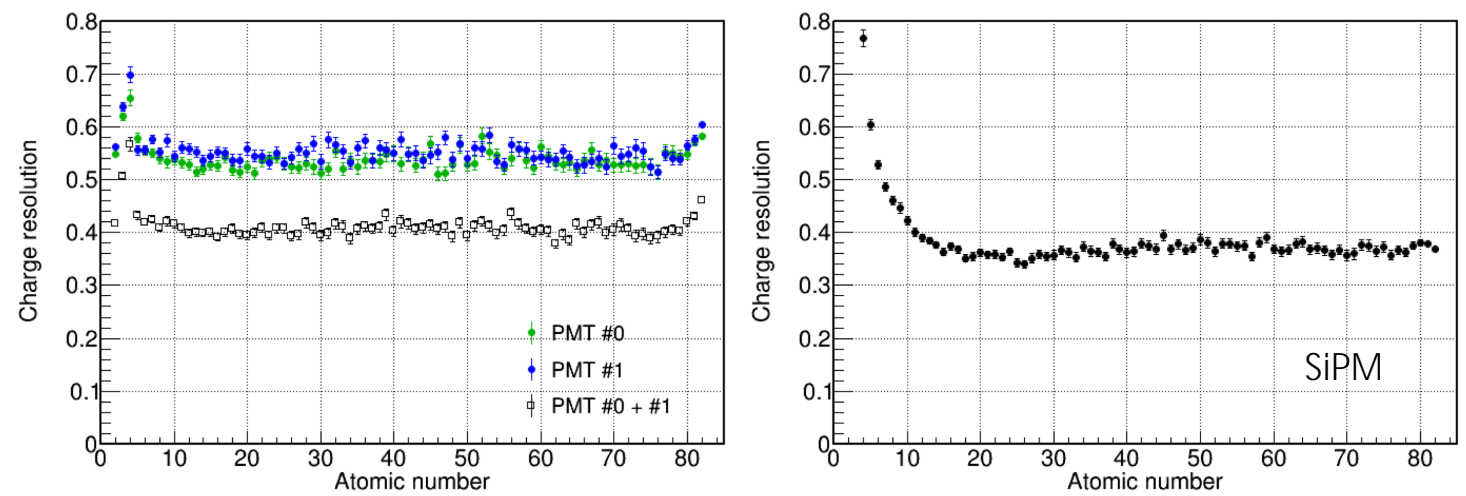

Figure 5: Comparison of the Charge Resolution of the R1924 PMTs and S14160 SiPMs

limited time we had for testing we were unable to determine how many of these events were heavy events and had to rely on beam statistics rather than our instrument trigger to determine the number of particles that passed through the SiPM.

In 2018 we returned with an improved setup for SiPM exposure. The SiPMs were mounted on a EPL SiPM board similar to the board in the lightboxes but designed to support 10 rather than 30 SiPMs. The SiPMs were then placed in the beam for a period of time and exposed to heavy elements. We were able to expose the SiPMs and measure the composition of particles using the other detectors in the test beam. We exposed two different $6 \mathrm{~mm} \times 6 \mathrm{~mm}$ SiPMs from Hammamatsu, the S13360-6050PE and the S14160-6050HS. In Table 1 we summarize the number of particles that 


\begin{tabular}{|l|l|l|l|l|l|l|}
\hline SiPM & $\begin{array}{l}\text { NIM } \\
\text { Trigger } \\
\text { Scaler }\end{array}$ & $\begin{array}{l}\text { Total } \\
\text { Recorded } \\
\text { Events }\end{array}$ & $\begin{array}{l}\text { Recorded } \\
\text { Events } \\
\text { Fe and above }\end{array}$ & $\begin{array}{l}\text { Events Fe and } \\
\text { heavier going } \\
\text { thru SiPM }\end{array}$ & $\begin{array}{l}\text { Recorded } \\
\text { Events } \\
\text { Pb and above }\end{array}$ & $\begin{array}{l}\text { Events Pb and } \\
\text { heavier going } \\
\text { thru SiPM }\end{array}$ \\
\hline S13360 & 3461073 & 2461075 & 731444 & 519668 & 106015 & 75320 \\
\hline S14160 & 2375934 & 1397031 & 286116 & 203276 & 7377 & 5241 \\
\hline
\end{tabular}

Table 1: Table showing the counts of particles passing through the beamline detector setup when the exposure box was in place. The Raw NIM tigger was set as described above and includes triggers during pedestal calibration runs. The total recorded events is the totally number of beamline events recorded taken the acquisition system during testing when the exposure box was in place. The recorded events with $\mathrm{Z}>26$ is based on a charge cut on the Caltech S1 silicon detector. The estimated number of events in the last column is taken based on taking the ratio of the area of the SiPM exposed in the beam divided by the beam area cross section.

the SiPMs were exposed to in our testing.

After the beam test we characterized the SiPMs exposed in the beam with unexposed SiPMs from Hammamatsu to examine if there were any significant variations in response. In Figure 4 we plot the IV curves of the exposed and unexposed S13360 and S14160 SiPM. The IV curve is generated by looking at the current drawn by a SiPM in a light tight enclosure and is a measure of the dark current of the photosensor. We note that in both cases the exposed SiPM draws a larger current but the difference in the current draw is very small, less than $<0.75 \%$ for either SiPMs over the tested voltage bias range.
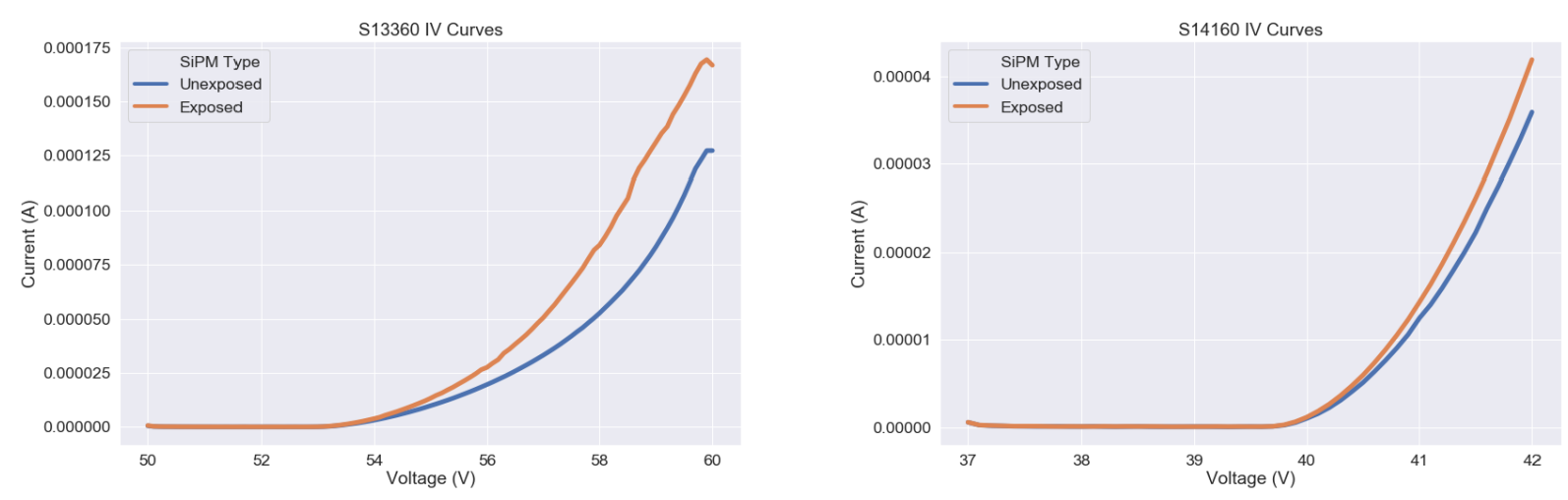

Figure 6: IV Plots of the unexposed and exposed Hammamatsu SiPM detectors.

In Figure 5 we plot the SiPM response to light from a small CsI(Na) crystal being stimulated by a Cs137 source. The bias voltage of the S13360 SiPM was set to 55V and the Bias Voltage of the S14160 SiPM was set to $40.7 \mathrm{~V}$ as recommended on the data sheet. The relative energy resolution of the unexposed S13360 SiPM photopeak (defined as FWHM/center) is $7.348 \%$ and the relative energy resolution of the exposed S13360 peak is $7.831 \%$. The relative energy resolution of the unexposed S14160 SiPM photopeak is $6.894 \%$ and the relative energy resolution of the exposed S14160 peak is $7.371 \%$. The change in resolution between the unexposed and exposed SiPMs is quite similar for both types of sensors. For the S13360 the energy resolution increased by $6.6 \%$, and for the $\mathrm{S} 14160$ the energy resolution increased by $6.9 \%$. 

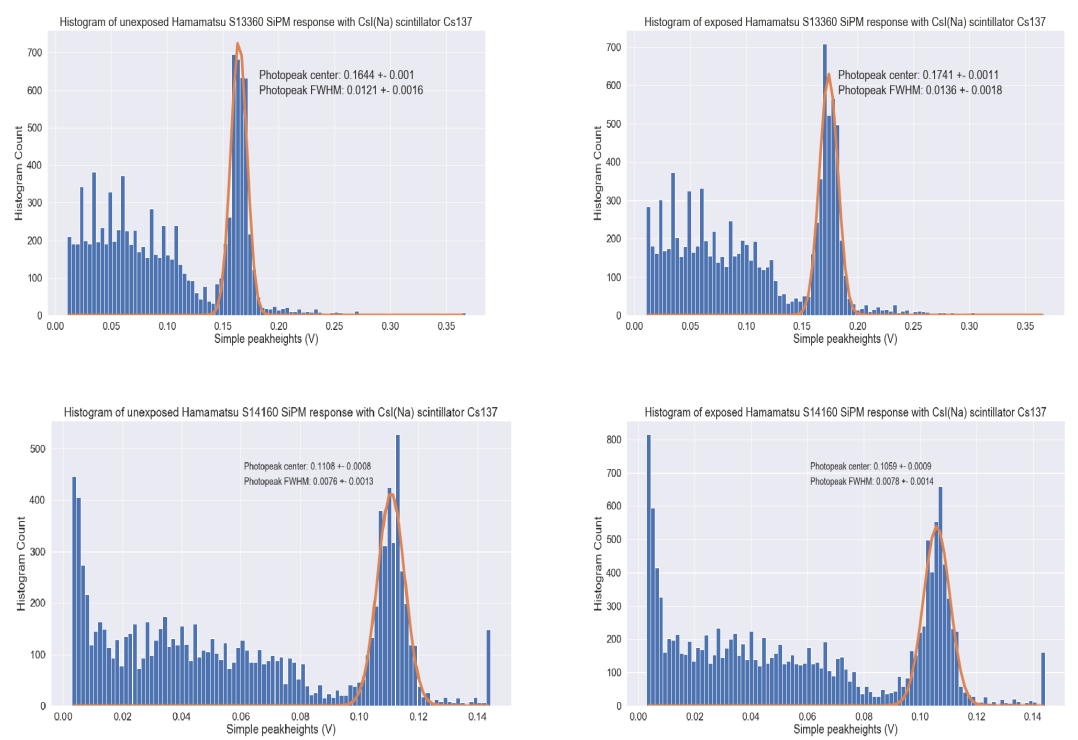

Figure 7: Histograms from Cs137 response test for the unexposed and exposed Hammamatsu SiPM photosensors.

\section{Conclusions}

SiPMs and PMTs were tested in a lead beam at CERN and both demonstrated they had sufficient dynamic range and charge resolution to measure individual elements from $Z=5$ to $Z=82$. The charge resolution of the S14160 SiPMs was the same, possibly slightly better than the R1924A PMTs used in this test. A heavy ion exposure test of S13360 and S14160 SiPMs was performed by putting SiPMs directly in the beam line. After exposure these sensors showed signs of slight performance degradation. The IV curves for each type of SiPMs showed an increase in dark current draw and the response peak for each type of SiPM showed an increase in peak location and width. The exposure was significantly greater than one would expect for a nominal space mission and the performance degradation was small suggesting heavy ion exposure is of minimal concern.

\section{References}

[1] Krizmanic et al., HNX/SuperTIGER Silicon Response to Nuclei in Lead Primary and Fragmented Test Beams, Proc. of 36rd ICRC Conference, Madison, United States (2019).

[2] Link et al., SiPM Use in Future Space Instruments, Proc. of 35rd ICRC Conference, Busan, South Korea (2017).

[3] Murphy, Ryan., Identifying the Origin of Galactic Cosmic Rays with the SuperTIGER Instrument, Ph.D Thesis, Washington University, St. Louis Missouri (2015).

[4] Musienko et al., Radiation Damage Studies of Silicon Photomultipliers for the CMS HCAL Phase 1 Upgrade, Nucl. Inst. Meth. Phys. Res. A 787, 319-322 (2015). 